\section{Linear Antenna Array Synthesis with Invasive Weed Optimization Algorithm}

\author{
Siddharth Pal ${ }^{1}$, Anniruddha Basak ${ }^{1}$, Swagatam Das ${ }^{1}$, and Ajith \\ Abraham $^{2}$ \\ ${ }^{1}$ Dept. of Electronics and Telecommunication Engg. , \\ Jadavpur University, Kolkata 700 032, India. \\ ${ }^{2}$ Norwegian University of Science and Technology, Norway \\ E-mail: sidd_pal2002@yahoo.com, aniruddha_ju_etce@yahoo.com, \\ swagatamdas19@yahoo.co.in, ajith.abraham@ieee.org
}

\begin{abstract}
Linear antenna array design is one of the most important electromagnetic optimization problems of current interest. This article describes the application of a recently developed metaheuristic algorithm, known as the Invasive Weed Optimization (IWO), to optimize the spacing between the elements of the linear array to produce a radiation pattern with minimum side lobe level and null placement control.The results of the IWO algorithm have been shown to meet or beat the results obtained using other state-of-the-art metaheuristics like the Genetic Algorithm (GA), Particle Swarm Optimization (PSO), Memetic Algorithms (MA), and Tabu Search (TS) in a statistically meaningful way.
\end{abstract}

Keywords: Antenna array, null control, invasive weeds optimization, side lobe suppression, metaheuristics.

\section{Introduction}

Antenna arrays play an important role in detecting and processing signals arriving from different directions. The goal in antenna array geometry synthesis is to determine the physical layout of the array that produces a radiation pattern that is closest to the desired pattern. The shape of the desired pattern can vary widely depending on the application. Many synthesis methods are concerned with suppressing the Side Lobe Level (SLL) while preserving the gain of the main beam [4]. Other methods deal with the null control to reduce the effects of interference and jamming. For the linear array geometry, this can be done by designing the spacing between the elements, while keeping a uniform excitation over the array aperture. Other methods of controlling the array pattern use non-uniform excitation and phased arrays [1].
It is well known that the classical derivative-based optimization techniques need a starting point that is reasonably close to the final solution, or they are likely to be stuck in a local minimum. If the initial values fall in a region of the solution space where all the local solutions are poor, a local search is limited to finding the best of these poor solutions. The computational drawbacks of existing numerical methods have forced the researchers all over the world to rely on metaheuristic algorithms founded on simulations of some natural phenomena to solve antenna problems. These algorithms use an objective function, optimization of which leads to the side lobe suppression and null control [5].

In recent past, the computational cost having been reduced almost dramatically, researchers all over the world are paying a considerable amount of attention towards bio-inspiration and bio-mimicry, for solving computational problems and constructing intelligent systems like autonomous robots. Following this tradition, in 2006, Mehrabian and Lucas proposed the Invasive Weed Optimization (IWO) [20], a derivative-free, metaheuristic algorithm, mimicking the ecological behavior of colonizing weeds. In this paper, IWO is used (for the first time, to the best of our knowledge) to optimize the spacing between the elements of the linear array to produce a radiation pattern with minimum SLL and null placement control. Three numerical instantiations of the design problem have been used to illustrate the application of the algorithm. Comparison with the results obtained with other best known metaheuristics like GA, PSO, TS, MA etc. reflect the superiority of IWO in a statistically significant fashion.

The rest of the paper is organized in the following way. A formulation of the array pattern synthesis as an optimization task has been discussed in Section 2. Section 3 provides a comprehensive overview of the IWO algorithm. Experimental settings have been discussed and the results have been presented in Section 4. Section 5 finally concludes the paper and unfolds a few future research issues. 


\section{Formulation of the Design Problem}

An antenna array is a configuration of individual radiating elements that are arranged in space and can be used to produce a directional radiation pattern. For a linear antenna array, let us assume that we have $2 N$ isotropic radiators placed symmetrically along the $x$-axis.

The array geometry is shown in Figure 1.

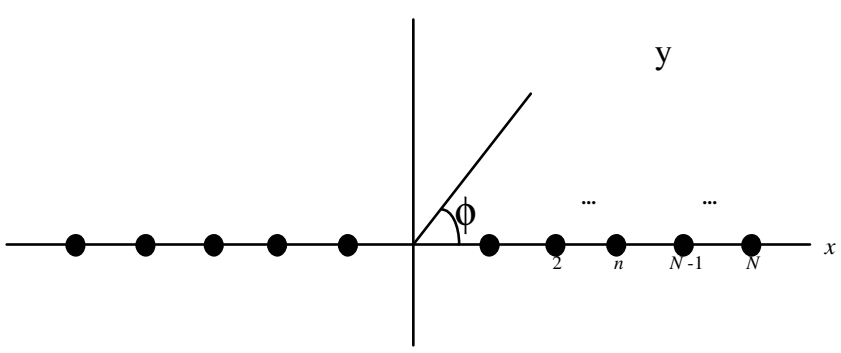

Fig 1: Symmetrically placed linear array 1

The array factor in the azimuth plane can be written as,

$A F(\phi)=2 \cdot \sum_{n=1}^{N} I_{n} \cdot \cos \left[k \cdot x_{n} \cdot \cos (\phi)+\varphi_{n}\right]$,

Where $\mathrm{k}$ is the wave number, and $I_{n}, \varphi_{n}$, and $x_{n}$ are, respectively excitation magnitude, phase and location of the $n$ th element. If we further assume a uniform excitation of amplitude and phase (that is $I_{n}=1$ and $\varphi_{n}=0$ for all elements), the array factor can be further simplified as:

$$
A F(\phi)=2 \cdot \sum_{n=1}^{N} \cos \left[k \cdot x_{n} \cdot \cos (\phi)\right]
$$

Now the statement of the problem, addressed here, simply reduces to: apply the IWO algorithm to find the locations $x_{n}$ of the array elements that will result in an array beam with minimum SLL and, if desired, nulls at specific directions.

For side lobe suppression, the objective function is:

$$
f_{1}=\sum_{i} \frac{1}{\Delta \phi_{i}} \int_{\phi_{l i}}^{\phi_{u i}}|A F(\phi)|^{2} \cdot d \phi
$$

And for null control we use:

$$
f_{2}=\sum_{k}\left|A F\left(\phi_{k}\right)\right|^{2}
$$

To minimize both of them we use a sum of (3) and (4) as our combined objective function of the IWO algorithm. Note that in (3), $\Delta \phi_{i}$ represents the bandwidth to suppress and $\Delta \phi=\phi_{u i}-\phi_{l i}$, and in (4) $\phi_{k}$ is the direction of the nulls.

\section{An Overview of IWO Algorithm}

Invasive Weed Optimization (IWO) is a meta-heuristic algorithm that mimics the colonizing behavior of weeds. The algorithm for IWO may be summarized as follows:

\section{Initialization:}

A finite number of weeds are initialized at the same element position of the conventional array which has a uniform spacing of $\lambda / 2$ between neighboring elements.

\section{Reproduction:}

Each member of the population is allowed to produce seeds depending on its own, as well as the colony's lowest and highest fitness, such that, the number of seeds produced by a weed increases linearly from lowest possible seed for a weed with worst fitness to the maximum no. of seeds for a plant with best fitness.

\section{Spatial distribution:}

The generated seeds are being randomly distributed over the $\mathrm{d}$ dimensional search space by normally distributed random numbers with mean equal to zero; but varying variance. This step ensures that the produced seeds will be generated around the parent weed, leading to a local search around each plant. However, the standard deviation (SD) of the random function is made to decrease over the iterations.

If $s d_{-} \max$ and $s d_{-} \min$ be the maximum and minimum standard deviation and if pow be a real no., then 
the standard deviation for a particular iteration may be given as in equation (5):

$$
s d_{\text {ITER }}=\left(\frac{\text { iter }_{\max }-\text { iter }}{\text { iter }_{\max }}\right)^{\text {pow }}\left(s d_{\max }-s d_{\min }\right)+s d_{\min }
$$

This step ensures that the probability of dropping a seed in a distant area decreases nonlinearly with iterations, which results in grouping fitter plants and elimination of inappropriate plants. Therefore, this is a selection mechanism of IWO.

\section{Competitive Exclusion:}

If a plant leaves no offspring then it would go extinct, otherwise they would take over the world. Thus, there is a need of some kind of competition between plants for limiting maximum number of plants in a colony. Initially, the plants in a colony will reproduce fast and all the produced plants will be included in the colony, until the number of plants in the colony reaches a maximum value $p o p_{\max }$. However, it is expected that by this time the fitter plants have reproduced more than undesirable plants. From then on, only the fittest plants, among the existing ones and the reproduced ones; are taken in the colony and the steps 1 to 4 are repeated until the maximum number of iterations has been reached, i.e. the colony size is fixed from thereon to pop $_{\max }$. This method is known as competitive exclusion and is also a selection procedure of IWO.

A flowchart of the whole optimization process has been shown in Figure 2

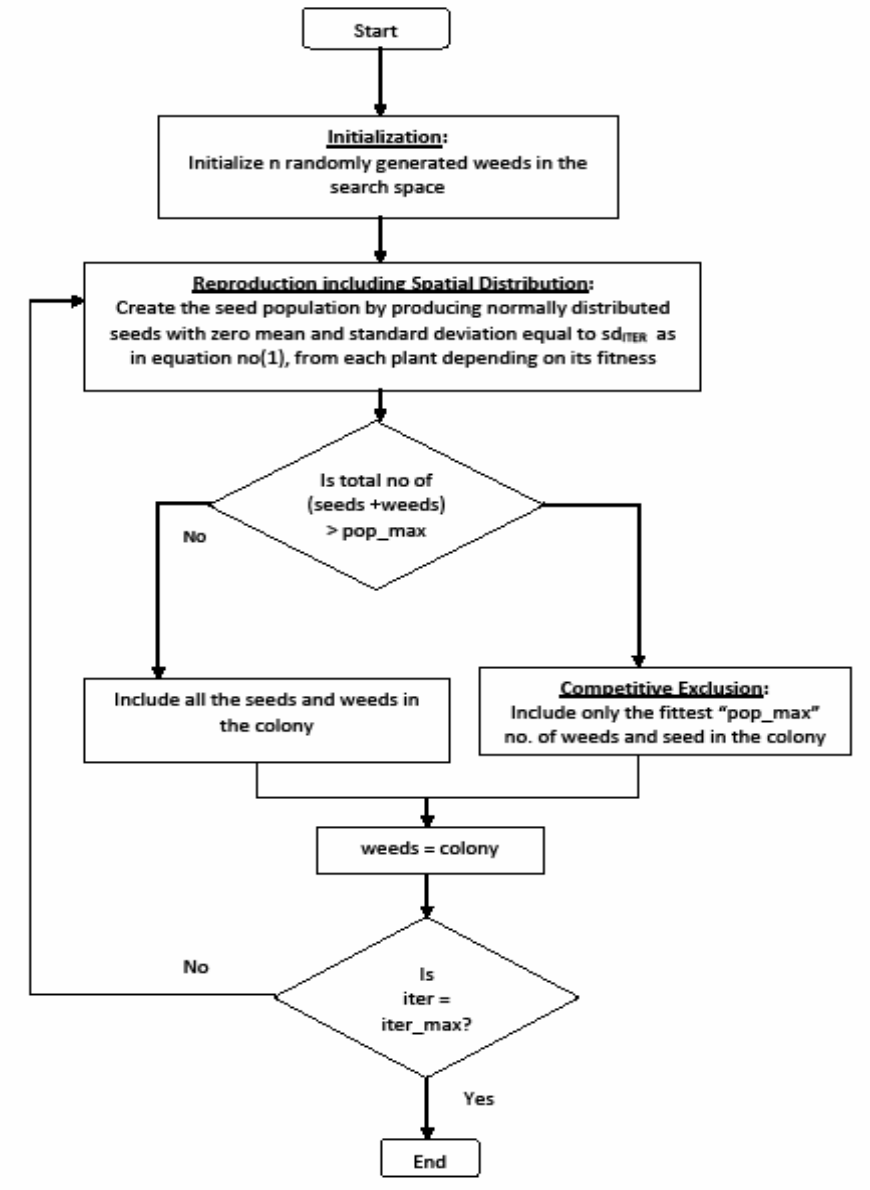

Fig. 2: A Flow-Chart Representation of the IWO Algorithm

\section{Experimental Results}

Here we have used Invasive Weed Colony Optimization technique for linear antenna design. The results have been compared with that of Genetic Algorithm (GA), Tabu Search Algorithm (TSA), Memetic Algorithm (MA) and Particle Swarm Optimization (PSO). For IWO we used the following parametric setup for all the design examples considered here: $s d_{\max }=0.1, s d_{\min }=10^{-5}$, initial number of plants $=$ $5^{*}$ dimensions, maximum number of seeds $=5$ and maximum plants $=20 *$ dimensions. Once set, no further hand tuning has been allowed for any of the parameter. For the competitor algorithms PSO, GA, MA, and TSA, we used the best possible parametric setup as explained in the relevant literatures [34, 36].In the first example IWO,GA,TSA, PSO, and MA were used to design 12 element array for minimum SLL in bands 
$\left[0^{\circ}, 82^{\circ}\right]$ and $\left[98^{\circ}, 180^{\circ}\right]$ and no null direction. Figure 3 shows the Gain versus Azimuth Angle plot. Table 4 shows the geometry of the linear array normalized to $\lambda / 2$

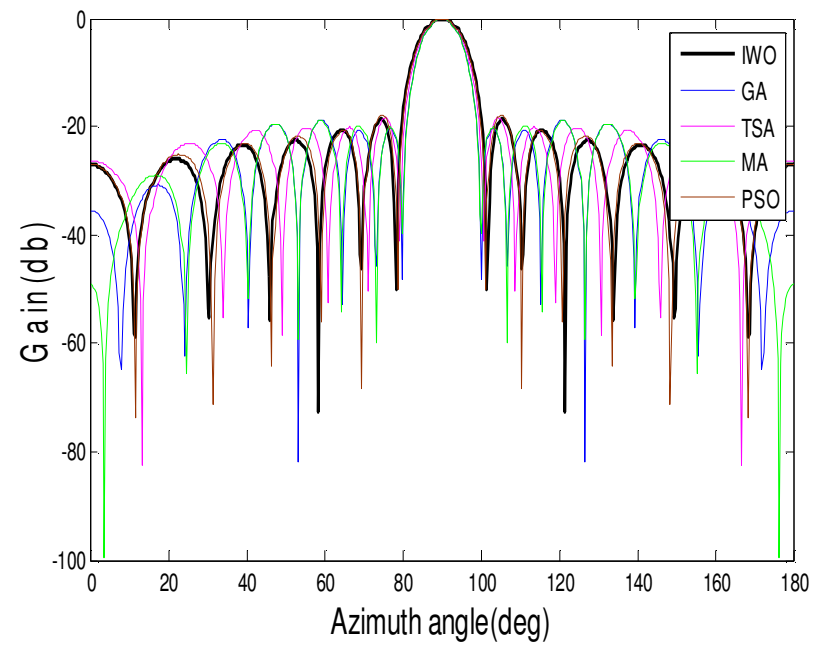

Fig. 3: Normalized patterns for 12 element array (example 1)

Table 1 shows the mean objective function values and standard deviations as obtained for the different optimization technique.A non-parametric statistical test called Wilcoxon's rank sum test for independent samples $[80,81]$ is conducted at the $5 \%$ significance level .

\begin{tabular}{|c|c|c|c|c|c|}
\hline Algorithm & IWO & GA & TSA & PSO & MA \\
\hline $\begin{array}{c}\text { Mean obj. } \\
\text { func. value }\end{array}$ & $\mathbf{0 . 0 0 8 7 6 7}$ & 0.009061 & 0.011219 & 0.010843 & 0.009196 \\
\hline Std. Dev. & $\mathbf{0 . 0 0 0 5 1 3}$ & 0.000681 & 0.000712 & 0.000319 & 0.000521 \\
\hline$P$-values & NA & $2.2917 \mathrm{e}-$ & $1.3493 \mathrm{e}-$ & $3.9034 \mathrm{e}-$ & $1.3493 \mathrm{e}-$ \\
& & 10 & 11 & 13 & 16 \\
\hline
\end{tabular}

Table 1: Statistical values pertaining to example 1

The second example has minimum SLL in bands $\left[0^{\circ}, 82^{\circ}\right]$ and $\left[98^{\circ}, 180^{\circ}\right]$ and null direction in $81^{\circ}$ and $99^{\circ}$. The array pattern from the IWO algorithm is shown in Figure 4, along with patterns obtained using other competitive metaheuristics. From Figure 4 it is evident that IWO has minimized SLL to the greatest extent and has a low gain value at the null directions as well. The P-values show that the difference between the mean objective function values obtained with IWO and any other competitive algorithm is statistically significant. In the third example, 26 element array has been designed for minimum SLL in bands $\left[0^{\circ}, 80^{\circ}\right]$ and $\left[100^{\circ}, 180^{\circ}\right]$ with nulls at $12^{\circ}, 60^{\circ}, 120^{\circ}$, and $168^{\circ}$.

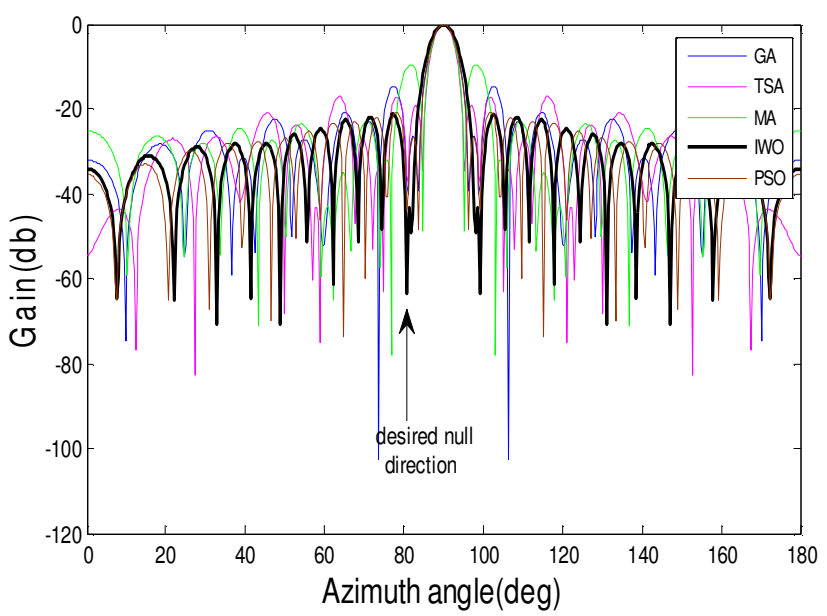

Fig.4: Normalized patterns for 22 element array(example 2)

\begin{tabular}{|c|c|c|c|c|c|}
\hline & IWO & GA & TSA & PSO & MA \\
\hline $\begin{array}{c}\text { Mean } \\
\text { fitness }\end{array}$ & $\mathbf{0 . 0 1 4 7 7 0}$ & 0.023662 & 0.021898 & 0.016755 & 0.021630 \\
\hline Std. Dev. & $\mathbf{0 . 0 0 1 0 7 2}$ & 0.001201 & 0.001109 & 0.001613 & 0.001331 \\
\hline$P$-values & NA & $6.1304 \mathrm{e}-12$ & $5.5647 \mathrm{e}-12$ & $9.6808 \mathrm{e}-15$ & $8.0040 \mathrm{e}-17$ \\
\hline
\end{tabular}

Table 2: Statistical values pertaining to example 2

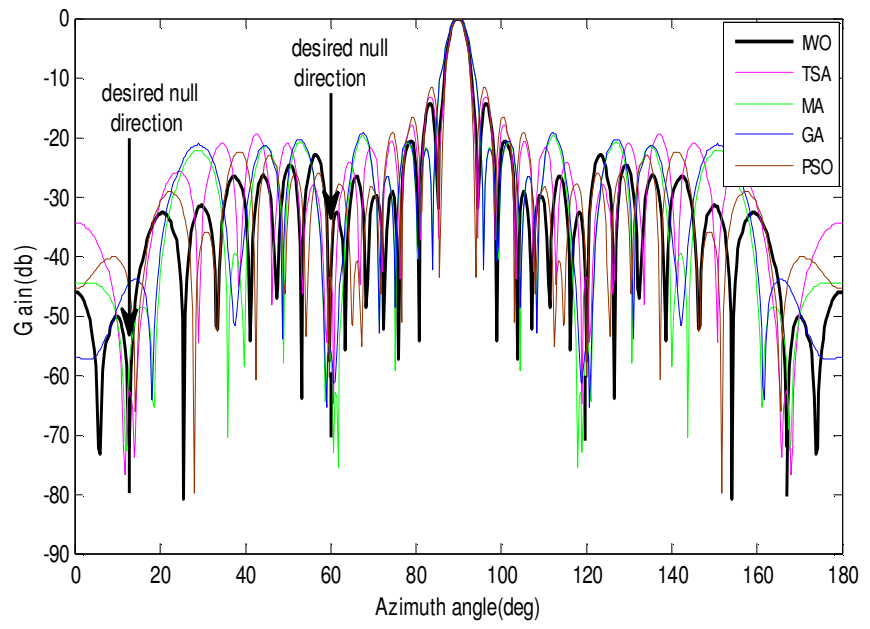

Fig.5:Normalized patterns for 26 element array(example 3) 


\begin{tabular}{|c|c|c|c|c|c|}
\hline Algorithms & IWO & GA & TSA & PSO & MA \\
\hline Mean fitness & $\mathbf{0 . 0 1 4 7 3 7}$ & 0.050279 & 0.061428 & 0.048131 & 0.027195 \\
\hline Std. Dev. & $\mathbf{0 . 0 0 1 4 2 5}$ & 0.003163 & 0.008221 & 0.003331 & 0.001109 \\
\hline$P$-Values & NA & $5.5647 \mathrm{e}-$ & $9.6808 \mathrm{e}-15$ & $4.0040 \mathrm{e}-$ & $6.1304 \mathrm{e}-$ \\
& & 12 & & 13 & 12 \\
& & & & & \\
\hline
\end{tabular}

Table 3: Statistical values pertaining to example 3

\begin{tabular}{|c|c|c|c|c|c|c|}
\hline IWO & \pm 0.0000 & \pm 1.330508 & \pm 1.815547 & \pm 2.912000 & \pm 3.823687 & \pm 5.100259 \\
\hline GA & \pm 0.506812 & \pm 1.261121 & \pm 2.288589 & \pm 3.191000 & \pm 4.561101 & \pm 5.999814 \\
\hline TSA & \pm 0.388182 & \pm 1.258812 & \pm 2.107651 & \pm 3.052561 & \pm 4.188121 & \pm 5.450781 \\
\hline PSO & \pm 0.247040 & \pm 1.310992 & \pm 1.936749 & \pm 2.971791 & \pm 3.927218 & \pm 5.180897 \\
\hline MA & \pm 0.481956 & \pm 1.278123 & \pm 2.288123 & \pm 3.235781 & \pm 4.602561 & \pm 5.999021 \\
\hline
\end{tabular}

Table 4: Geometry of the 12 element linear array normalized with respect to $\lambda / 2$ (median solution of 50 runs)

\begin{tabular}{|c|c|c|c|c|c|c|c|c|c|c|c|c|c|}
\hline IWO & \pm 0.4483 & \pm 1.488 & \pm 2.275 & \pm 3.279 & \pm 4.254 & \pm 5.326 & \pm 6.161 & \pm 7.168 & \pm 8.046 & \pm 9.091 & \pm 9.928 & \pm 11.24 & \pm 12.68 \\
\hline TSA & \pm 0.5311 & \pm 1.491 & \pm 2.468 & \pm 3.445 & \pm 4.524 & \pm 5.501 & \pm 6.491 & \pm 7.268 & \pm 8.498 & \pm 9.514 & \pm 10.27 & \pm 11.44 & \pm 12.42 \\
\hline
\end{tabular}

Table 5: Geometry of the 22 element linear array normalized with respect to $\lambda / 2$ (median solution of 50 runs)

\begin{tabular}{|c|c|c|c|c|c|c|c|c|c|c|c|}
\hline IWO & \pm 0.3501 & \pm 1.3643 & \pm 1.939 & \pm 2.795 & \pm 3.637 & \pm 4.800 & \pm 5.949 & \pm 7.459 & \pm 8.582 & \pm 9.667 & \pm 11.000 \\
\hline GA & \pm 0.0002 & \pm 1.061 & \pm 1.486 & \pm 2.419 & \pm 3.386 & \pm 4.285 & \pm 5.407 & \pm 6.846 & \pm 8.042 & \pm 9.136 & \pm 10.398 \\
\hline TSA & \pm 0.6982 & \pm 1.071 & \pm 2.485 & \pm 2.541 & \pm 4.148 & \pm 5.479 & \pm 6.480 & \pm 7.573 & \pm 8.714 & \pm 10.211 & \pm 11.64 \\
\hline PSO & \pm 0.3006 & \pm 1.177 & \pm 1.855 & \pm 2.685 & \pm 3.524 & \pm 4.428 & \pm 5.468 & \pm 6.580 & \pm 7.953 & \pm 9.552 & \pm 11.00 \\
\hline MA & \pm 0.8113 & \pm 2.273 & \pm 3.157 & \pm 3.948 & \pm 4.770 & \pm 5.411 & \pm 6.432 & \pm 6.934 & \pm 7.896 & \pm 8.712 & \pm 10.124 \\
\hline
\end{tabular}

Table 6: Geometry of the 26 element linear array normalized with respect to $\lambda / 2$ (median solution of 50 runs)

Figure 5 shows that IWO has successfully minimized both the sidelobe level and in the required null directions.Tables 3 and 6 present similar results as Tables 1 and 4 but now for design example 3.The convergence characteristics of the five algorithms over design problem 3 have been shown in Figure 6 in terms of the mean objective function value (in logarithmic scale) of the median run of each algorithm versus the number of FEs.

Table 3 shows that IWO can yield statistically significantly better final accuracy than all its competitors. Figure 6 indicates that not only does IWO yield the most accurate results for nearly the design problem, but it does so consuming the least amount of computational time. Note that we omitted the convergence graph for the first two design problems in order to save space and also in consideration of the fact that these graphs show a similar trend.

\section{Conclusion}

This paper illustrated the use of the IWO algorithm in the synthesis of linear array geometry for the purpose of suppressed side lobes and null placement in certain directions. IWO was successfully used to optimize the locations of array elements to exhibit an array pattern with either suppressed sidelobes, null placement in certain directions, or both.

In each of these cases, the IWO algorithm easily achieved the optimization goal, beating four other state-of-the-art optimization techniques in a statistically significant fashion. Future research may focus on achieving more control of the array pattern by using the IWO algorithm to optimize, not only the location, but also the excitation amplitude and phase of each element in the array, and exploring other array geometries. 


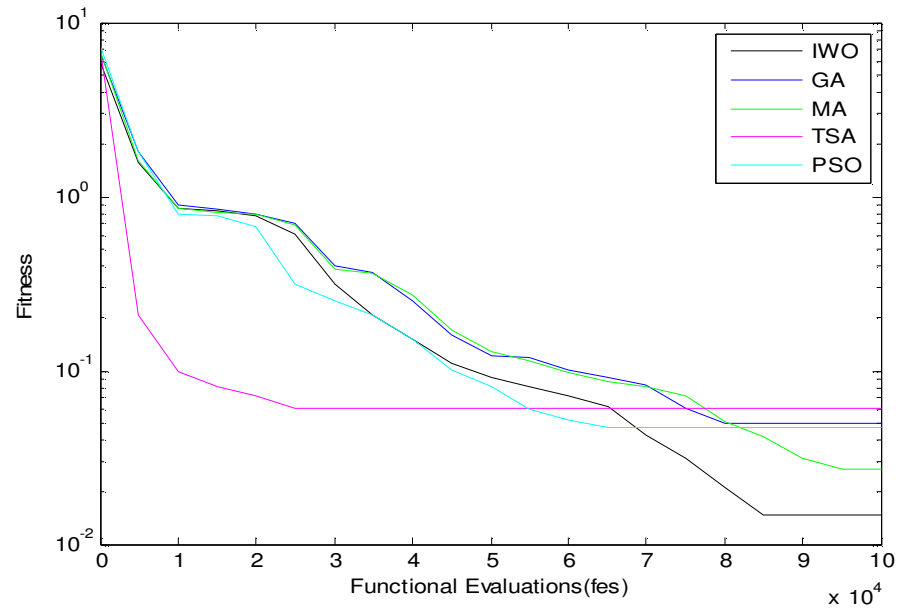

Fig. 6: Convergence curve of the fitness value corresponding to example 3

As a metaheuristic algorithm, IWO will most likely be an increasingly attractive alternative, in the electromagnetics and antennas community, to other evolutionary algorithms such as GAs and PSO. Compared to genetic algorithms and simulated annealing, IWO is much easier to understand and implement, minimizes the need for problem-dependent parameter tuning, and requires minimum mathematical preprocessing.

\section{References}

1. Handbook of Antennas in Wireless Communications, L. C. Godara, Ed., CRC, Boca Raton, FL, 2002.

2. O.M. Bucci, D. D’Elia, G. Mazzarella, and G. Panatiello, Antenna pattern synthesis: A new general approach. Proc. IEEE, 82: 358-371, 1994.

3. Y. Rahmat-Samii and E. Michielssen Eds., Electromagnetic Optimization by Genetic Algorithms. New York: Wiley, 1999.

4. H. Lebret and S. Boyd, "Antenna array pattern synthesis via convex optimization," IEEE Transactions on Signal Processing, Vol. 45, No. 3, March 1997.

5. M. M. Khodier and C. G. Christodoulou, "Linear array geometry synthesis with minimum side lobe level and null control using particle swarm optimization," IEEE Transactions on Antennas and Propagation, Vol. 53, No. 8, August 2005.
6. J. H. Holland, Adaptation in Natural and Artificial Systems, University of Michigan Press, Ann Harbor, 1975.

7. T. Bäck, D. Fogel, Z. Michalewicz, Handbook of Evolutionary Computation, Oxford Univ. Press, 1997.

8. A.E. Eiben and J.E. Smith, Introduction to Evolutionary Computing, Springer, 2003.

9. S. Kirkpatrik, C. Gelatt, and M. Vecchi, Optimization by Simulated Annealing. Science, 220: 671-680, 1983.

10. F. Glover and M. Laguna, Tabu Search, Kluwer, Norwell, MA, 1997.

11. G. Taguchi, S. Chowdhury, and Y.Wu, Taguchi's Quality Engineering Handbook. New York: Wiley, 2005.

12. Y.-S. Ong, and A. J. Keane, "Meta-lamarckian learning in memetic algorithms," IEEE Transactions on Evolutionary Computation, vol. 8, no. 2, pp. 99-110, 2004.

13. J. Kennedy and R. Eberhart, "Particle swarm optimization," Proc. IEEE Int. conf. Neural Networks, pp.1942-1948, 1995.

14. J. Kennedy, R. C. Eberhart, and Y. Shi, Swarm Intelligence, Morgan Kaufmann, San Francisco, CA, 2001.

15. A. Udina, N. M. Martin, and L. C. Jain, "Linear antenna array optimization by genetic means," Third International Conference on Knowledge-Based Intelligent Information Engineering Systems Adelaide, Australia, Sept. 1999.

16. Y. Cengiz and H. Tokat, Linear Antenna Array Design With use of Genetic, Memetic and Tabu Search Optimization Algorithms,

Progress In Electromagnetics Research (PIER) C, Vol. 1, 63$72,2008$.

17. W-C. Weng, F. Yang, and A. Z. Elsherbeni, Linear Antenna Array Synthesis Using Taguchi's Method: A Novel Optimization Technique in Electromagnetics, IEEE Transactions on Antennas and Propagation, Vol. 55, No. 3, pp. 723 - 730, March 2007.

18. F. J. Ares-Pena, A. Rodriguez-Gonzalez, E. Villanueva-Lopez, and S. R. Rengarajan, "Genetic algorithms in the design and optimization of antenna array patterns," IEEE Transactions on Antennas and Propagation, vol. 47, pp. 506-510, Mar. 1999.

19. Y. B. Tian and J. Qian, "Improve the performance of a linear array by changing the spaces among array elements in terms of genetic algorithm," IEEE Transactions on Antennas and Propagation., vol. 53, pp. 2226-2230, Jul. 2005.

20. A. R. Mehrabian and C. Lucas, "A novel numerical optimization algorithm inspired from weed colonization," Ecological Informatics, vol. 1, pp. 355-366, 2006. 
21. A. R. Mehrabian, A. Yousefi-Koma, "Optimal positioning of piezoelectric actuators on a smart fin using bio-inspired algorithms", Aerospace Science and Technology, 2007, vol. 11, pp.174-182.

22. H. Sepehri Rad, C. Lucas, "A recommender system based on invasive weed optimization algorithm", IEEE Congress on Evolutionary Computation, CEC 2007, Sept. 2007, pp. 42974304.

23. A. R. Mallahzadeh, H. Oraizi, and Z. Davoodi-Rad, "Application of the Invasive Weed Optimization Technique For Antenna Configurations", Progress In Electromagnetics Research PIER 79, 137-150, 2008.

24. A. R. Mallahzadeh, S. Es'haghi, and A. Alipour, "Design of an EShaped Mimo Antenna Using IWO Algorithm for Wireless Application at $5.8 \mathrm{GHz}$ ", Progress In Electromagnetics Research, PIER 90, 187 - 203, 2009.

25. X. Zhang, Y. Wang, G. Cui, Y. Niu, and J. Xu, “Application of a novel IWO to the design of encoding sequences for DNA computing”, Comput. Math. Appl. 57, pp. 2001-2008, Jun, 2009.

26. A. R. Mallahzadeh, S. Es'haghi, and H. R. Hassani, Compact Uarray MIMO antenna designs using IWO algorithm, International Journal of RF and Microwave Computer-Aided Engineering, Wiley-InterSscience, DOI: 10.1002/mmce.20379, Jul, 2009.

27. B.D. Van Veen, and K.M. Buckley, "Beamforming: A versatile approach to spatial filtering", IEEE Acoust. Speech Signal Process. Mag., 5: 4-24, 1988. 